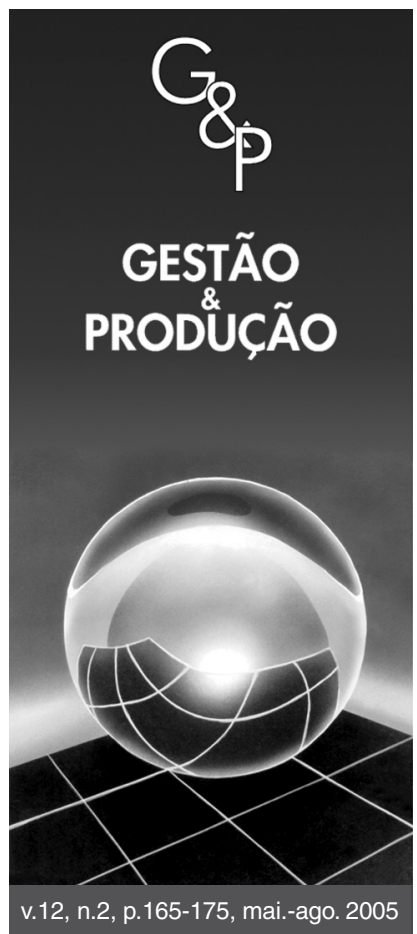

\title{
MICRO-NEGOCIOS ASOCIATIVOS CAMPESINOS: ANÁLISIS ECONÓMICO DE UN SISTEMA DE PRODUCCIÓN OVINA, REGIÓN DEL MAULE, CHILE
}

\author{
Germán Lobos Andrade \\ Escuela de Ingeniería Comercial, Facultad de Ciencias Empresariales, \\ Universidad de Talca, Casilla 721, Talca, Chile. \\ e-mail: globos@utalca.cl
}

Resumen

El objetivo de esta investigación fue evaluar la rentabilidad económica de un sistema de producción ovina para un grupo de 20 productores agropecuarios, cuyos predios se encuentran localizados en la zona de secano interior de la provincia de Linares, Región del Maule, Chile. El trabajo en terreno se realizó durante el periodo noviembre del 2002 a junio 2003, el cual permitió caracterizar a los productores en cuatro grupos homogéneos entre sí, en función del número de cabezas del rebaño ovino. Para estimar la rentabilidad económica del sistema propuesto, bajo distintos escenarios de precios de venta, se usó el Valor Actual Neto (VAN), la Tasa Interna de Retorno (TIR) y el Índice de Valor Actual Neto (IVAN). El valor promedio del rebaño ovino fue estimado entre 0,26 a \$1,45 millones y el valor promedio de la tierra entre 6,3 y \$12,8 millones, dependiendo del tamaño del predio agrícola (hectáreas). Para el escenario normal de precios, se obtuvo un VAN (10,8\%) de \$4,12 millones, la TIR se estimó en 14,5\% y el IVAN en 0,37. La principal conclusión sugiere que el emprendimiento asociativo a micro-escala puede contribuir a mejorar las condiciones de vida de los pequeños productores.

Palabras clave: pequeños propietarios, rentabilidad, rebaño ovino, predio agrícola.

\section{Introducción}

Las micro-empresas son unidades económicas generadoras de ingresos que se desarrollan en hogares al borde de la subsistencia, que emplean sólo al empresario y el valor del capital de explotación fluctúa entre 11.000 y 14.000 dólares (León y Schreiner, 1994). En el sector agropecuario el término micro-escala se refiere a pequeños productores que operan con bajos niveles de inversión, con muy baja capacidad de reinversión y escaso uso de mano de obra, los mercados para sus productos son básicamente locales, las estructuras de administración son simples, y donde normalmente se opera en un solo predio (Young, 1995). En Chile existen 318 pequeñas y micro-empresas rurales, de las cuales 238 son agrícolas y 80 son no agrícolas (Monares y Bustamante, 2004). Estos autores consideran que la diversificación es una estrategia efectiva para disminuir los riesgos inherentes a la producción agrícola, el cual tiende a ser más elevado en las pequeñas unidades de producción vinculadas al mercado.

Según el Censo Nacional Agropecuario 1996/1997 las existencias de ganado ovino en Chile sumaron 3,7 millones de cabezas en 1997, las cuales se redujeron a 3,4 millones de cabezas el año 2002; alrededor de un 52\% de dicho inventario se concentra en la Región de Magallanes, mientras que un $42 \%$ se encuentra localizado entre la Región de O’Higgins y Aysén. Durante el periodo 1997 a 
2002, el beneficio de ganado ovino pasó de 639.233 a 729.850 cabezas, la producción de carne en vara sólo aumentó de 9.811 a 9.857 t, y las exportaciones de carne ovina se incrementaron de 3.304 a 4.296 t (INE, 2002).

A nivel internacional, diversos estudios sugieren que la cantidad demandada por un tipo de carne no varía significativamente frente a un cambio en el precio de la misma carne (demanda relativamente inelástica con respecto al precio-propio), la demanda por un tipo de carne no cambia significativamente frente a una variación en el precio de otro tipo de carne (demanda inelástica con respecto al precio-cruzado) y que los distintos tipos de carnes son bienes normales (elasticidad ingreso positiva y menor que 1) o superiores (elasticidad ingreso positiva y mayor que 1). Algunos estudios son los de Hayes et al. (1991) para carne de bovinos, ovinos, cerdo, pollo y pescados; Rosson et al. (1993) para carne de ternera, cerdo, pollo, carnero y cordero; Kaabia et al., (2001) para carnes en general, y carne de pollo, cerdo y pescados; y Reed et al. (2003) para carnes en general, y carne de cerdo y pollo.

En Chile el consumo per cápita de carne ovina se ha mantenido relativamente estable desde el año 1999, en torno a $0,4 \mathrm{~kg} \mathrm{hab}^{-1}$. Según datos de ODEPA (2004), en términos reales, la variación anual de los precios promedio en los mercados mayoristas ha sido de $2 \%$ (carne de cordero), $1,4 \%$ (carne de oveja) y $-1 \%$ (carne de ovino).

En la zona costera de la provincia de Linares se ha desarrollado durante años una intensa actividad agrícola sobre el recurso suelo. Según Avendaño e Imbarach (2002), la mayoría de los suelos son de secano y el sistema productivo se basa en una combinación de cultivos (trigo) y ganado (ovinos y bovinos). La producción ovina se basa en las razas Suffolk-Down y Hampshire-Down y sus cruzas, y en el uso pastoral de praderas naturales anuales de baja calidad (Figueroa, 1986) y con escasa producción (Fernández et al., 2004; Avendaño et al., 2004), siendo la disponibilidad de alimento la principal limitante del desempeño productivo tanto de ovinos como de bovinos (Novoa, 1989). Además, el sistema tradicional de explotación pecuaria del secano interior dista bastante de las potencialidades del sector (Avendaño e Imbarach, 2002).

El objetivo de esta investigación fue evaluar la rentabilidad económica de un sistema de producción ovina para un grupo de 20 productores agropecuarios, cuyos predios se encuentran localizados en la zona de secano interior de las comunas de Parral y Retiro, provincia de

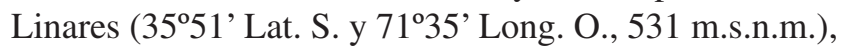
Región del Maule, Chile. Los productores se encuentran formalmente agrupados en una micro-empresa asociativa campesina. Se espera que este proyecto constituya la base del aprendizaje para un mejor manejo del rebaño ovino y contribuya a mejorar los niveles de ingresos de los productores y sus familias.

\section{Materiales y métodos}

\subsection{Caracterización de los predios agrope- cuarios}

Los datos fueron recolectados durante el periodo noviembre de 2002 a junio de 2003. Los productores fueron caracterizados en cuatro grupos relativamente homogéneos entre sí de acuerdo al número total de cabezas de ganado ovino (Tabla 1), de acuerdo al esquema de clasificación de productores lecheros propuesto por Díaz y Williamson (1998). Dentro del ganado ovino se incluyeron corderos, ovejas, carneros y borregas.

- Micro-productores: En este grupo se incluyeron 9 productores que poseen en promedio 13 cabezas de ganado ovino. La edad promedio de los productores es de 50 años. En este segmento se observa un $55,6 \%$ de propiedad sobre la tierra, el más bajo de todo el universo de productores considerado, visualizándose también un alto porcentaje de tierra en arriendo (33\%). La superficie promedio apta para cultivo es de 20,8 ha, de las cuales 18,9 ha son destinadas al cultivo de trigo y arroz. El valor promedio del rebaño ovino alcanza a $\$ 0,26 \mathrm{mi}-$ llones y el valor promedio de la tierra es de $\$ 6,3 \mathrm{mi}-$ llones por productor. Lo anterior se explica porque la actividad de los micro productores es relativamente más intensiva en cultivos agrícolas como fuente principal de ingresos para la familia.

- Muy pequeños productores: Se incluyeron 4 productores, que poseen en promedio 35 cabezas de ganado ovino. La edad promedio de los productores es de 70 años. Todos los predios están localizados en la comuna de Retiro. En este grupo se observa un 79,7\% de propiedad sobre la tierra, y un bajo porcentaje de tierra en arriendo $(16 \%)$. La superficie promedio apta para cultivo es de 27,3 ha, de las cuales 25 ha son destinadas al cultivo de trigo, arroz y chacras. El valor promedio del rebaño ovino alcanza a $\$ 0,71$ millones y el valor promedio de la tierra es de $\$ 7,2$ millones por productor. En este caso se observó una mayor diversificación en los cultivos agrícolas.

- Pequeños productores: Se consideraron 3 productores que poseen en promedio 56 cabezas de ganado ovino. La edad promedio de los productores es de 64 años. En este grupo se observa un $66,7 \%$ de propiedad sobre la tierra, y también un alto porcentaje de tierra en arriendo (33\%). La superficie promedio apta para cultivo es de 22 ha, de las cuales 18,3 ha son destinadas al cultivo de trigo, arroz, chacras y cebada. El valor promedio del rebaño ovino alcanza a $\$ 1,1$ millones y el valor promedio de la tierra es de \$5,7 millones por productor. En este caso también se observó una mayor diversificación en los cultivos agrícolas. 
Tabla 1. Caracterización promedio de los predios agropecuarios (valores en \$).

Table 1. Average features of farms (values in Chilean \$).

\begin{tabular}{cccc}
$\begin{array}{c}\text { Micro } \\
\text { productores }\end{array}$ & $\begin{array}{c}\text { Muy } \\
\text { pequeños } \\
\text { productores }\end{array}$ & $\begin{array}{c}\text { Pequeños } \\
\text { productores }\end{array}$ & $\begin{array}{c}\text { Medianos } \\
\text { productores }\end{array}$ \\
\hline & & & \\
\hline
\end{tabular}

Productores y localización de los predios:

Número de productores

Edad promedio de los socios (años)

\begin{tabular}{rr}
9 & 4 \\
50 & 70 \\
3 & 0 \\
6 & 4 \\
\hline
\end{tabular}

Número de predi

Stock de animales:

Total de ganado ovino (cabezas)

Promedio de cabezas ovinas por productor

Stock total de ganado bovino (cabezas)

Promedio de cabezas bovinas por productor

Stock total de equinos (cabezas)

Promedio de cabezas equinas por productor

Predios con asesoría veterinaria

Tenencia de la tierra (ha):

Superficie total

Número de productores dueños de la tierra

Promedio de propiedad de la tierra

Superficie total promedio por productor

Superficie total propia

Superficie propia promedio por productor

Superficie en arriendo

Superficie en mediería

Uso del suelo agrícola (ha):

Superficie total apta para cultivos

Superficie promedio por productor

Superficie total con riego

Superficie con riego promedio por productor

Productores con asesoría agronómica

Valorización del ganado ovino y la tierra (\$):

Valor total ganado ovino

Valor promedio por productor

Valor total de la tierra propia

Valor promedio por productor

or

50

\begin{tabular}{rr}
3 & 4 \\
64 & 50 \\
2 & 1 \\
1 & 3 \\
\hline
\end{tabular}

\begin{tabular}{rrrr}
18 & 141 & 167 & 290 \\
13 & 35 & 56 & 73 \\
17 & 33 & 24 & 25 \\
13 & 8 & 8 & 6 \\
81 & 16 & 16 & 22 \\
9 & 4 & 5 & 6 \\
$00 \%$ & $100 \%$ & $100 \%$ & $100 \%$ \\
\hline
\end{tabular}

(100\%

\begin{tabular}{cccc}
500 & 169 & 205 & 256 \\
5 & 4 & 2 & 4 \\
$55,6 \%$ & $79,7 \%$ & $66,7 \%$ & $100 \%$ \\
55,6 & 42,3 & 68,3 & 63,9 \\
284 & 143 & 85 & 256 \\
31,6 & 35,8 & 28,3 & 63,9 \\
186 & 20 & 120 & 0 \\
30 & 6 & 0 & 0 \\
187 & & & \\
20,8 & 109 & 66 & 93 \\
170 & 27,3 & 22,0 & 23,3 \\
18,9 & 100 & 55 & 91 \\
$100 \%$ & 25,0 & 18,3 & 22,8 \\
& $100 \%$ & $100 \%$ & $100 \%$ \\
\hline
\end{tabular}

El cuadro presenta las características más relevantes de los predios agropecuarios según el tipo de productor: micro, muy pequeños, pequeños y medianos productores.

- Medianos productores: Se incluyeron 4 productores que poseen en promedio 73 cabezas de ganado ovino. La edad promedio de los productores es de 50 años. Todos los productores son dueños del $100 \%$ de la tierra, lo que les permite tener un mayor nivel de autonomía e independencia. La superficie promedio apta para cultivo es de 23,3 ha, de las cuales 22,8 ha son destinadas al cultivo de trigo, arroz, chacras, cebada y pequeñas extensiones de viñedos. El valor promedio del rebaño ovino alcanza a $\$ 1,5$ millones y el valor promedio de la tierra es de
\$12,8 millones por productor. En este caso no sólo se observó aún una mayor diversificación en los cultivos agrícolas, sino que también una mayor incorporación de cultivos y plantaciones de mayor valor agregado.

Durante el periodo de estudio, todos los productores contaron con asesoría veterinaria y agronómica permanente, la cual fue financiada con recursos del Programa de Asesorías Técnicas (SAT) para la pequeña agricultura familiar campesina provenientes del Instituto de Desarrollo Agropecuario (INDAP). 


\subsection{Diagnóstico estratégico de la micro-em- presa}

Con el objeto de encontrar la mejor relación posible entre las tendencias que se perciben en el medio y el potencial de la micro-empresa, se realizó un análisis de la posición estratégica de ésta. Para ello se usó la metodología FODA (Fortalezas, Oportunidades, Debilidades, Amenazas), la cual entrega un marco conceptual para analizar sistemáticamente la interrelación entre las amenazas y oportunidades externas con las debilidades y fortalezas internas de una organización (Koontz y Weihrich, 1998).

\subsection{Coeficientes técnicos del sistema ovino}

Los coeficientes técnicos fueron obtenidos a partir de los modelos teórico-prácticos desarrollados en sucesivas investigaciones para sistemas ovinos en la zona del secano interior de la Región del Maule (Avendaño et al., 1978; Avendaño et al., 1994; Avendaño et al., 1996; Avendaño e Imbarach, 2002). El tamaño del proyecto fue diseñado como un sistema semiextensivo de 100 ovejas de la raza doble propósito Suffolk-Down, 4 carneros (1 por cada 25 ovejas) y 26 borregas. Se consideró una superficie total de pradera natural ocupada de 131 ha, distribuida en 2 potreros de 17,7 y 89,3 ha para pastoreo continuo diferido de ovejas, 1 potrero de 4 ha para carneros y 1 potrero de 20 ha para borregas (Avendaño et al., 1978). La carga animal se estimó en 1 oveja ha ${ }^{-1}$ año $^{-1}$ (Avendaño e Imbarach, 2002), equivalente a $0,2 \mathrm{UA} \mathrm{ha}^{-1} \mathrm{año}^{-1}$. Los principales índices productivos del sistema propuesto se presentan en la Tabla 2.

Como recurso alimenticio básico se consideró la pradera anual de secano (Avendaño et al., 1994; Avendaño et al., 1996), para la cual se usó una producción promedio de MS entre 1.000-1.200 $\mathrm{kg} \mathrm{ha}^{-1} \mathrm{año}^{-1}$ (Avendaño et al., 1978). Para la obtención de forraje suplementario de invierno se incluyó el establecimiento de 1,2 ha de pradera permanente, compuesta por trébol subterráneo (Trifolium subterraneum) - falaris (Phalaris tuberosa) - ballica australiana o wimmera (Lolium rigidum var. wimmera). Además, para todos los animales se consideró un suplemento de una mezcla de harina de huesos y sal común, en una proporción $2: 1$, respectivamente (Avendaño et al., 1978).

Por otra parte, el sistema propuesto contempla las siguientes construcciones y cercos: galpón (superficie de $20 \mathrm{~m}^{2}$ ), corral (superficie de $100 \mathrm{~m}^{2}$ ), baño tipo pileta (capacidad de $3.000 \mathrm{~L}$ ), comederos tipo canoa con protección (largo de $3 \mathrm{~m}$ ), habitación para el ovejero construida en material ligero (superficie de $60 \mathrm{~m}^{2}$ ) y cercos límites o exteriores (extensión de $2.314 \mathrm{~m}$ ) e interiores (extensión de $2.310 \mathrm{~m}$ ).

La producción total de carne del sistema ovino se estimó en $3.799 \mathrm{~kg}$ año $^{-1}$, distribuida en $2.305,6 \mathrm{~kg}$ año-1 de carne de cordero, 1.336,2 $\mathrm{kg}^{2}$ año ${ }^{-1}$ de carne de ovejas de
Tabla 2. Índices productivos del sistema ovino. Table 2. Productive indexes of semi extensive sheep system.

\begin{tabular}{|c|c|}
\hline Índice & Valor \\
\hline Carga animal anual $\left(\text { ovejas ha }{ }^{-1}\right)^{(1)}$ & 1,0 \\
\hline Superficie total del sistema (ha) ${ }^{(2)}$ & 132,2 \\
\hline Superficie total de pradera natural ocupada (ha) ${ }^{(2)}$ & 131,0 \\
\hline Superficie total de pradera permanente (ha) ${ }^{(2)}$ & 1,2 \\
\hline Ovejas vientres (cabezas) ${ }^{(1)}$ & 100,0 \\
\hline Reemplazo anual de vientres $(\%)^{(1)}$ & 20,0 \\
\hline Reemplazo anual de machos $(\%)^{(1)}$ & 25,0 \\
\hline $\begin{array}{l}\text { Parición (corderos nacidos vivos en base a ovejas } \\
\text { paridas) }(\%)^{(1)}\end{array}$ & 106,0 \\
\hline Ovejas secas (en base a ovejas encastadas) $(\%)^{(1)}$ & 8,0 \\
\hline Mortalidad corderos entre nacimiento y destete $(\%)^{(1)}$ & 4,6 \\
\hline Mortalidad ovejas (\%) ${ }^{(3)}$ & 7,3 \\
\hline Mortalidad borregas $(\%)^{(3)}$ & 6,7 \\
\hline Peso corderos al destete $(\mathrm{kg})^{(3)}$ & 34,2 \\
\hline Peso oveja de rechazo $(\mathrm{kg})^{(3)}$ & 54,5 \\
\hline Peso borrega de 2 dientes al encaste $(\mathrm{kg})^{(2)}$ & 48,0 \\
\hline Peso carnero de rechazo $(\mathrm{kg})^{(2)}$ & 85,0 \\
\hline Producción anual de carne de cordero $\left(\mathrm{kg} \mathrm{ha}^{-1}\right)^{(3)}$ & 17,6 \\
\hline Producción anual de carne de oveja $\left(\mathrm{kg} \mathrm{ha}^{-1}\right)^{(3)}$ & 10,2 \\
\hline Producción anual de carne carnero $\left(\mathrm{kg} \mathrm{ha}^{-1}\right)^{(3)}$ & 1,2 \\
\hline Producción anual de lana $\left(\mathrm{kg} \mathrm{ha}^{-1}\right)^{(3)}$ & 1,9 \\
\hline
\end{tabular}

(1) Para un sistema extensivo mejorado (Avendaño e Imbarach, 2002);

(2) Avendaño et al., 1978;

(3) Corresponden a los valores medios de las temporadas 1981/82 a 1986/87 (Avendaño et al., 1996).

desecho y $157,2 \mathrm{~kg}$ año ${ }^{-1}$ de carne de carneros de desecho. La producción total de lana se estimó en $248,9 \mathrm{~kg}$ año ${ }^{-1}$, equivalente a $2,5 \mathrm{~kg}_{\text {oveja }}{ }^{-1}$ año $^{-1}$ (Tabla 2).

\subsection{Evaluación económica del proyecto}

Para estimar la rentabilidad económica del sistema se calcularon los siguientes indicadores (Fontaine, 1997; Díaz y Williamson, 1998; Sapag y Sapag, 2000): Valor Actual Neto (VAN), Valor Anual Equivalente (VAE), Tasa Interna de Retorno (TIR), Índice de Valor Actual Neto (IVAN) y Período de Recuperación de Capital (PRC). En los costos totales de producción se incluyeron los costos directos, los gastos generales y de administración, y otros gastos. Para efectos del cálculo de impuestos, se utilizó la tasa de impuesto a la renta de $1^{\text {a }}$ Categoría actualmente vigente (17\%). Dado que se asumió que los productores son dueños de la tierra, desde el punto de vista económico, se incluyó el costo alternativo del arriendo del terreno agrícola.

Se consideró un horizonte de evaluación de 10 años. La proyección de los ingresos anuales se realizó a partir de la producción anual estimada en el estudio de (Avendaño et al., 1996). El precio de venta de la carne de 
cordero, oveja de desecho y carnero de desecho se estimó como el precio promedio real, en moneda de agosto de 2004, pagado en los mercados mayoristas durante el período septiembre 1999 a agosto 2004 para las carnes de cordero, oveja y ovinos, respectivamente (ODEPA, 2004). En el caso del precio de la lana, se utilizó el precio promedio recibido por los micro-productores durante la última temporada. Sin embargo, dada la complejidad de los mercados de la carne y lana de ovinos, se simularon los siguientes escenarios de precio de venta:

Escenario normal $(n): p_{i}^{n}=\dot{p}$

Escenario optimista (o): $\mathrm{p}_{\mathrm{i}}^{\circ}=\dot{\mathrm{p}}_{\mathrm{i}}+\sigma_{\mathrm{i}}$

Escenario pesimista (p): $\mathrm{p}_{\mathrm{i}}^{\mathrm{p}}=\dot{\mathrm{p}}_{\mathrm{i}}-\sigma_{\mathrm{i}}$

Donde $\mathrm{p}_{\mathrm{i}}$ es el precio del producto i de cada escenario, $\dot{\mathrm{p}}_{\mathrm{i}}$ es el precio promedio real del producto i durante el periodo septiembre 1999 a agosto 2004, y ó es la desviación estándar de la serie de precios reales del producto i durante el periodo septiembre 1999 a agosto 2004. Todos los precios están expresados en $\$ \mathrm{~kg}^{-1}$. Además, $\mathrm{i}=1, \ldots, 4$ donde $\mathrm{i}=1$ es carne de cordero, $\mathrm{i}=2$ es carne de oveja, $i=3$ es carne de ovino, e $i=4$ es lana. Para este último caso se consideraron los valores de la última temporada.

Los precios utilizados en la evaluación del proyecto fueron los siguientes:

Escenario normal: $\mathrm{p}_{1}^{\mathrm{n}}=1.380,4 ; \quad \mathrm{p}_{2}^{\mathrm{n}}=774,2 ;$ $\mathrm{p}_{3}^{\mathrm{n}}=1.175,1 ; \mathrm{p}_{4}^{\mathrm{n}}=250,5$

Escenario optimista: $\mathrm{p}_{1}^{\mathrm{o}}=1.491,8 ; \quad \mathrm{p}_{2}^{\mathrm{o}}=891,2 ;$ $\mathrm{p}_{3}^{o}=1.232,7 ; \mathrm{p}_{4}^{\mathrm{o}}=260,3$

Escenario pesimista: $\mathrm{p}_{1}^{\mathrm{p}}=1.268,9 ; \quad \mathrm{p}_{2}^{\mathrm{p}}=657,1 ;$ $\mathrm{p}_{3}^{\mathrm{p}}=1.117,4 ; \mathrm{p}_{4}^{\mathrm{p}}=240,3$

Para la valorización de las inversiones y costos esperados se utilizaron los precios vigentes a agosto del 2004 , sin impuestos. Todos los activos fueron depreciados de acuerdo al método de depreciación lineal, es decir, al valor de la inversión se le descontó el valor residual y el resultado se dividió por la vida útil de cada activo. En el caso del ganado, el Servicio de Impuestos Internos (SII, 2002), mediante Resolución N $\mathrm{N}^{\circ} 43$ del 26 de diciembre de 2002, autorizó la depreciación de la inversión en ovejas, sólo para efectos tributarios, considerando una vida útil normal de 5 años. De acuerdo a la legislación chilena, la depreciación puede ser tratada como costo sólo para efectos de rebajar el pago de impuestos. Dado que la depreciación no constituye un flujo de efectivo, una vez que se obtiene la utilidad después de impuestos debe sumarse el monto de la depreciación para compensar el ajuste contable y no considerarla en el flujo neto de caja. El capital de trabajo se estimó como un $50 \%$ de los costos directos del primer año de operación. Los costos de producción fueron estructurados de acuerdo a la clasificación de costos para empresas agropecuarias propuesto por Vargas (2004). En el costo de la mano de obra se incluyeron las leyes sociales, vacaciones proporcionales, seguro de desempleo y gratificaciones. Para las estimaciones se utilizó una tasa de descuento del 10,8\%, estimada por Lobos et al. (2001) para la evaluación de proyectos de lecherías localizadas en la Región del Maule.

Se aplicó el modelo multidimensional de sensibilización (Sapag y Sapag, 2000) para medir el efecto de los cambios en algunas variables sobre la rentabilidad del proyecto. Dado que los cambios en los precios de los insumos no fueron relevantes sobre la rentabilidad, se incluyó el efecto de los cambios en la producción, superficie total de pradera natural ocupada y la tasa de descuento, además de los precios de venta de los productos.

\section{Resultados y discusión}

La caracterización de los predios agropecuarios sugiere que los productores se encuentran en el rango de micro-empresas que desarrollan actividades a micro-escala, de acuerdo a lo reportado por la literatura (León y Schreiner, 1994; Young, 1995). Desde el punto de vista de la posición estratégica de la micro-empresa, la matriz FODA permitió identificar como principales fortalezas la experiencia de los agricultores en la producción ovina, poseer una cartera de clientes en el mercado local y el hecho de contar con asesoría técnica permanente de los organismos de apoyo a la agricultura. Sin embargo, las principales debilidades detectadas fueron la falta de definición de la Misión y objetivos de la organización, la escasez de capital de trabajo y la falta de capacidad de gestión individual. Por otra parte, como oportunidades del ambiente externo a la organización se visualizaron mayores oportunidades de mercado para la carne ovina originadas por la firma de nuevos Tratados de Libre Comercio, con una demanda por carnes en aumento. No obstante, se observaron amenazas derivadas de la calidad de la carne producida, la existencia de una variada cantidad de sustitutos cercanos para este producto, y la pérdida de mercado para la lana de oveja, debido básicamente a la mayor difusión de productos sintéticos y de mejor calidad, y la mayor competencia de grandes productores-acopiadores, tales como Australia, Nueva Zelanda y China. La demanda por carnes en aumento y la existencia de sustitutos cercanos es consistente con los resultados de las elasticidades ingreso y precio-cruzada reportados por la literatura (Hayes et al., 1991; Rosson et al., 1993; Kaabia et al., 2001; Reed et al., 2003).

Con respecto al sistema ovino propuesto, la inversión total fue estimada en $\$ 21,97$ millones (Tabla 3), e inclu- 
Tabla 3. Flujos de inversiones y costos estimados (\$).

Table 3. Investment and cost flows estimated (Chilean \$).

\begin{tabular}{|c|c|c|c|c|}
\hline Inversiones & Año 0 & Año 1 & Año 2-9 & Año 10 \\
\hline Terreno agrícola $^{(1)}$ & 0 & & & \\
\hline Instalaciones $\operatorname{cercos}^{(2)}$ & - 3.471.699 & & & 1.735 .850 \\
\hline Pradera suplementaria (trébol-falaris-ballica) ${ }^{(3)}$ & -339.422 & & & 0 \\
\hline Construcciones $^{(4)}$ & -3.326 .000 & & & 2.197.800 \\
\hline Animales $^{(5)}$ & -4.342 .080 & & & 3.729 .000 \\
\hline Vehículo con equipo de frío(6) & -9.000 .000 & & & 900.000 \\
\hline Capital de trabajo & -1.489 .156 & & & 1.489 .156 \\
\hline Total inversiones & - 21.968.358 & & & 10.051 .806 \\
\hline Costos directos & Año 0 & Año 1 & Año 2-9 & Año 10 \\
\hline \multicolumn{5}{|l|}{ 1. Alimentación } \\
\hline Mantención anual de praderas & & 81.424 & 81.424 & 81.424 \\
\hline Mantención anual de cercos & & 26.775 & 26.775 & 26.775 \\
\hline Conservación de forraje (ensilaje) & & 137.100 & 137.100 & 137.100 \\
\hline Suplementación mineral & & 349.973 & 349.973 & 349.973 \\
\hline Sub-total de alimentación & & 595.273 & 595.273 & 595.273 \\
\hline \multicolumn{5}{|l|}{ 2. Mano de obra } \\
\hline Permanente (ovejero) & & 1.428 .000 & 1.428 .000 & 1.428 .000 \\
\hline Esquila & & 39.000 & 39.000 & 39.000 \\
\hline Sanidad animal & & 26.000 & 26.000 & 26.000 \\
\hline Sub-total de mano de obra & & 1.493 .000 & 1.493 .000 & 1.493 .000 \\
\hline \multicolumn{5}{|l|}{ 3. Vacunas y medicamentos } \\
\hline Antiparasitarios & & 75.700 & 75.700 & 75.700 \\
\hline Vacunas & & 60.840 & 60.840 & 60.840 \\
\hline Sub-total vacunas y medicamentos & & 136.540 & 136.540 & 136.540 \\
\hline \multicolumn{5}{|l|}{ 4. Reemplazos } \\
\hline Reemplazo carneros & & 38.500 & 38.500 & 38.500 \\
\hline Reemplazo ovejas & & 715.000 & 715.000 & 715.000 \\
\hline Sub-total reemplazos & & 753.500 & 753.500 & 753.500 \\
\hline \multicolumn{5}{|l|}{ 5. Transporte } \\
\hline $\mathrm{MPO}^{(7)}$ a planta faenadora & & 480.000 & 480.000 & 480.000 \\
\hline Total costos directos & & 3.458.313 & 3.458 .313 & 3.458.313 \\
\hline
\end{tabular}

(1) Como costo del terreno se incluyó el costo alternativo del arriendo del suelo agrícola.

(2) Incluye cercos exteriores e interiores con una vida útil de 20 años y un valor residual del 50\%. La depreciación anual se estimó en $\$ 86.792$.

(3) Se consideró una vida útil de 5 años y un valor residual del 0\%. La depreciación anual se estimó en \$ 67.884.

(4) Incluye galpón y corrales (vida útil 20 años y valor residual del 60\%), baño tipo pileta (vida útil 30 años y valor residual del $70 \%$ ), comederos (vida útil 10 años y valor residual del 0\%), y habitación ovejero (vida útil 40 años y valor residual del 80\%). La depreciación anual se estimó en \$53.740.

${ }^{(5)}$ Para la valorización de los animales se consideraron los siguientes precios de compra ( $\$ \mathrm{cab}^{-1}$ ): 35.750 (ovejas), 38.500 (carneros) y 23.580 (borregas). El tamaño del proyecto fue diseñado para 100 ovejas, 4 carneros y 26 borregas, por lo cual la inversión inicial en animales se estimó en \$ 4.342.080, de los cuales \$ 3.575.000 corresponden a la inversión en ovejas, con una vida útil normal de 5 años. La depreciación anual de las ovejas se estimó en \$715.000.

(6) Se consideró una vida útil de 10 años y un valor residual del 10\%. La depreciación anual se estimó en \$810.000.

(7) Módulo Productivo Ovino.

ye: instalaciones y cercos $(15,8 \%)$, establecimiento de la pradera suplementaria $(1,5 \%)$, construcciones $(15,1 \%)$, animales (19,8\%), vehículo con equipo de frío (41\%), y capital de trabajo $(6,8 \%)$. Los costos directos esperados se estimaron en \$ 3,46 millones anuales (Tabla 3), distri- buidos en alimentación (17,2\%), mano de obra $(43,2 \%)$, vacunas y medicamentos $(3,9 \%)$, reemplazos $(21,8 \%)$ y transporte $(13,9 \%)$.

Los ingresos anuales esperados fueron estimados en $\$ 8,25$ millones bajo el escenario normal de precios, 
$\$ 8,94$ millones bajo el escenario optimista y $\$ 7,57 \mathrm{mi}-$ llones bajo el escenario pesimista. La venta de lana representa entre un 6,7 y 7,3\% de los ingresos totales (Tabla 4). Para los escenarios definidos de precios de venta de productos, la estimación de los flujos netos de caja se presentan en la Tabla 5.

Los resultados de la evaluación económica del sistema ovino y el análisis de sensibilidad se presenta en la Tabla 6. El VAN estimado fue de \$ 4,12 millones (escenario normal de precios), $\$ 7,49$ millones (escenario optimista) y $\$ 0,76$ millones (escenario pesimista). La TIR varió en un rango entre 11,5 y 17,4\% bajo los escenario pesimista y optimista, respectivamente. El IVAN obtenido fue menor que 1 en todos los casos. El PRC se estimó en 6 y 10 años bajo el escenario optimista y normal, respectivamente. En el escenario pesimista no se recupera el capital invertido en el proyecto durante el período de evaluación del mismo. Con respecto al escenario normal de precios, los cambios en la producción afectan fuertemente los resultados de la evaluación económica, aunque en ambos casos el proyecto continúa siendo rentable. Lo mismo ocurre en el caso en que se disminuye la tasa de descuento al $8 \%$. Por otra parte, cuando se reduce la superficie total de pradera natural ocupada a 118 ha, lo cual

Tabla 4. Flujos de ingresos estimados.

Table 4. Income flows estimated.

\begin{tabular}{|c|c|c|c|c|}
\hline Descripción & Año 0 & Año 1 & Año 2-9 & Año 10 \\
\hline \multicolumn{5}{|l|}{ Producción (kg año) } \\
\hline Carne de cordero & & $2.305,6$ & $2.305,6$ & $2.305,6$ \\
\hline Carne de oveja & & $1.336,2$ & $1.336,2$ & $1.336,2$ \\
\hline Carne de ovino & & $1.175,1$ & $1.175,1$ & $1.175,1$ \\
\hline Lana de ovinos & & 248,9 & 248,9 & 248,9 \\
\hline \multicolumn{5}{|l|}{ Precios escenario normal $(\$ \mathrm{~kg})$} \\
\hline Carne de cordero & & $1.380,4$ & $1.380,4$ & $1.380,4$ \\
\hline Carne de oveja & & 774,2 & 774,2 & 774,2 \\
\hline Carne de ovino & & $1.175,1$ & $1.175,1$ & $1.175,1$ \\
\hline Lana de ovinos & & 250,5 & 250,5 & 250,5 \\
\hline \multicolumn{5}{|l|}{ Precios escenario optimista $(\$ \mathrm{~kg})$} \\
\hline Carne de cordero & & $1.491,8$ & $1.491,8$ & $1.491,8$ \\
\hline Carne de oveja & & 891,2 & 891,2 & 891,2 \\
\hline Carne de ovino & & $1.232,7$ & $1.232,7$ & $1.232,7$ \\
\hline Lana de ovinos & & 260,5 & 260,5 & 260,5 \\
\hline \multicolumn{5}{|l|}{ Precios escenario pesimista $(\$ \mathrm{~kg})$} \\
\hline Carne de cordero & & $1.268,9$ & $1.268,9$ & $1.268,9$ \\
\hline Carne de oveja & & 657,1 & 657,1 & 657,1 \\
\hline Carne de ovino & & $1.117,4$ & $1.117,4$ & $1.117,4$ \\
\hline Lana de ovinos & & 240,5 & 240,5 & 240,5 \\
\hline Ingresos escenario normal (\$) & & 8.254 .314 & 8.254 .314 & 8.254 .314 \\
\hline Carne de cordero & & 3.182 .535 & 3.182 .535 & 3.182 .535 \\
\hline Carne de oveja & & 1.784 .938 & 1.784 .938 & 1.784 .938 \\
\hline Carne de ovino & & 2.709 .289 & 2.709.289 & 2.709 .289 \\
\hline Lana de ovinos & & 577.553 & 577.553 & 577.553 \\
\hline Ingresos escenario optimista (\$) & & 8.937.179 & 8.937.179 & 8.937.179 \\
\hline Carne de cordero & & 3.439 .558 & 3.439 .558 & 3.439 .558 \\
\hline Carne de oveja & & 2.054 .804 & 2.054 .804 & 2.054 .804 \\
\hline Carne de ovino & & 2.842.208 & 2.842 .208 & 2.842 .208 \\
\hline Lana de ovinos & & 600.609 & 600.609 & 600.609 \\
\hline Ingresos escenario pesimista (\$) & & 7.571.449 & 7.571 .449 & 7.571.449 \\
\hline Carne de cordero & & 2.925 .512 & 2.925 .512 & 2.925 .512 \\
\hline Carne de oveja & & 1.515 .072 & 1.515 .072 & 1.515 .072 \\
\hline Carne de ovino & & 2.576 .369 & 2.576 .369 & 2.576 .369 \\
\hline Lana de ovinos & & 554.497 & 554.497 & 554.497 \\
\hline
\end{tabular}


Tabla 5. Flujos netos de caja estimados (\$).

Table 5. Net cash flows estimated (Chilean \$).

\begin{tabular}{|c|c|c|c|c|}
\hline Escenario normal & Año 0 & Año 1 & Año 2-9 & Año 10 \\
\hline Inversiones & -21.968 .358 & & & \\
\hline Valor residual & & & & 10.051 .806 \\
\hline Ingresos por venta de carne y lana & & 8.254 .314 & 8.254 .314 & 8.254 .314 \\
\hline Costos directos & & -3.458 .313 & -3.458 .313 & - 3.458.313 \\
\hline Depreciación de activos y ovejas & & -1.733 .417 & - 1.733 .417 & - 1.733 .417 \\
\hline MARGEN BRUTO & & 3.062 .585 & 3.062 .585 & 3.062 .585 \\
\hline Gastos generales & & 143.600 & 143.600 & 143.600 \\
\hline Gastos de administración y ventas & & 150.000 & 150.000 & 150.000 \\
\hline Otros gastos ( $7 \%$ de costos directos) & & 242.082 & 242.082 & 242.082 \\
\hline UTILIDAD ANTES DE IMPUESTOS & & 2.526 .903 & 2.526 .903 & 2.526 .903 \\
\hline Pago de impuestos $(17 \%)$ & & -429.573 & -429.573 & -429.573 \\
\hline UTILIDAD DESPUÉS DE IMPUESTOS & & 2.097 .329 & 2.097.329 & 2.097 .329 \\
\hline Depreciación de activos y ovejas & & 1.733 .417 & 1.733 .417 & 1.733 .417 \\
\hline Arriendo terreno agrícola & -37.800 & - 37.800 & - 37.800 & -37.800 \\
\hline Flujo Neto de Caja & -22.006 .158 & 3.792 .946 & 3.792 .946 & 13.844.752 \\
\hline Escenario optimista & Año 0 & Año 1 & Año 2-9 & Año 10 \\
\hline Inversiones & - 21.968.358 & & & \\
\hline Valor residual & & & & 10.051 .806 \\
\hline Ingresos por venta de carne y lana & & 8.937 .179 & 8.937 .179 & 8.937 .179 \\
\hline Costos directos & & -3.458 .313 & -3.458 .313 & - 3.458.313 \\
\hline Depreciación de activos y ovejas & & - 1.733.417 & - 1.733.417 & - 1.733.417 \\
\hline MARGEN BRUTO & & 3.745 .450 & 3.745 .450 & 3.745 .450 \\
\hline Gastos generales & & 143.600 & 143.600 & 143.600 \\
\hline Gastos de administración y ventas & & 150.000 & 150.000 & 150.000 \\
\hline Otros gastos ( $7 \%$ de costos directos) & & 242.082 & 242.082 & 242.082 \\
\hline UTILIDAD ANTES DE IMPUESTOS & & 3.209 .768 & 3.209 .768 & 3.209 .768 \\
\hline Pago de impuestos (17\%) & & -545.661 & -545.661 & -545.661 \\
\hline UTILIDAD DESPUÉS DE IMPUESTOS & & 2.664 .107 & 2.664 .107 & 2.664 .107 \\
\hline Depreciación de activos y ovejas & & 1.733 .417 & 1.733 .417 & 1.733 .417 \\
\hline Arriendo terreno agrícola & -37.800 & -37.800 & -37.800 & -37.800 \\
\hline Flujo Neto de Caja & - 22.006.158 & 4.359.724 & 4.359.724 & 14.411.530 \\
\hline Escenario pesimista & Año 0 & Año 1 & Año 2-9 & Año 10 \\
\hline Inversiones & - 21.968.358 & & & \\
\hline Valor residual & & & & 10.051 .806 \\
\hline Ingresos por venta de carne y lana & & 7.571 .449 & 7.571 .449 & 7.571 .449 \\
\hline Costos directos & & -3.458 .313 & -3.458 .313 & - 3.458.313 \\
\hline Depreciación de activos y ovejas & & - 1.733.417 & - 1.733.417 & - 1.733.417 \\
\hline MARGEN BRUTO & & 2.379 .719 & 2.379 .719 & 2.379 .719 \\
\hline Gastos generales & & 143.600 & 143.600 & 143.600 \\
\hline Gastos de administración y ventas & & 150.000 & 150.000 & 150.000 \\
\hline Otros gastos ( $7 \%$ de costos directos) & & 242.082 & 242.082 & 242.082 \\
\hline UTILIDAD ANTES DE IMPUESTOS & & 1.844 .038 & 1.844 .038 & 1.844 .038 \\
\hline Pago de impuestos (17\%) & & -313.486 & -313.486 & -313.486 \\
\hline UTILIDAD DESPUÉS DE IMPUESTOS & & 1.530 .551 & 1.530 .551 & 1.530 .551 \\
\hline Depreciación de activos y ovejas & & 1.733 .417 & 1.733 .417 & 1.733 .417 \\
\hline Arriendo terreno agrícola & -37.800 & - 37.800 & -37.800 & -37.800 \\
\hline Flujo Neto de Caja & -22.006 .158 & 3.226 .168 & 3.226 .168 & 13.277.974 \\
\hline
\end{tabular}


Tabla 6. Análisis de sensibilidad: indicadores de rentabilidad del sistema ovino.

Table 6. Sensibility analysis: profitability indicators of sheep system.

$\begin{array}{ccc}\text { Descripción } & \text { Escenario de precios de venta de productos y de producción } \\ \text { (rendimiento) } & \begin{array}{c}\text { Tasa de } \\ \text { descuento } \\ \text { más baja }\end{array} & \begin{array}{c}\text { Superficie } \\ \text { total menor }\end{array}\end{array}$

\section{Parámetros:}

Precios $\left(\$ \mathrm{~kg}^{-1}\right)$

Producción $(\mathrm{kg} \text { año-1 })^{(1)}$

Superficie total (ha)

Tasa de descuento $(\%)^{(3)}$

Indicadores de rentabilidad:

Valor ac tual neto $(\$)$

Valor anual equivalente $(\$)$

Tasa interna de retorno $(\%)$

Índice de valor actual neto (\$)

Período recuperación capital

$\begin{array}{rr}\text { Normal } & \text { Optimista } \\ \text { Normal } & \text { Normal } \\ 131 & 131 \\ 10,8 & 10,8 \\ & \\ 4.124 .469 & 7.490 .535 \\ 694.478 & 1.261 .256 \\ 14,49 & 17,44 \\ 0,19 & 0,34\end{array}$

10 años

\begin{tabular}{cr} 
Pesimista & \multicolumn{2}{l}{ Normal } \\
Normal & \multicolumn{2}{c}{ Alta } \\
131 & 131 \\
10,8 & 10,8
\end{tabular}

Normal
Baja
131
10,8
1.276 .294
214.902
11,95
0,06
10 años

$\begin{array}{rr}6.972 .645 & 1.276 .294 \\ 1.174 .054 & 214.902 \\ 16,99 & 11,95 \\ 0,32 & 0,06 \\ 8 \text { años } & 10 \text { años }\end{array}$

Normal
Normal
131
$8,0^{(4)}$

8.100 .751
1.207 .251
14,49
0,37
9 años

Normal

Normal

$118^{(2)}$

10,8

55.647

9.370

10,85

0,16

(1) El escenario de producción alta (baja) asume un valor del 7\% superior (inferior) con respecto al escenario normal.

(2) Se asume que la superficie total de pradera natural ocupada se reduce en un $10 \%$ con respecto al escenario normal.

(3) Fuente: Estimada por Lobos et al. (2001) para estimar la rentabilidad de distintas lecherías localizadas en la Región del Maule.

(4) Corresponde a la tasa de descuento sugerida por INDAP para la evaluación de proyectos presentados anualmente al Concurso de Modernización de la Agricultura Familiar Campesina.

representa una disminución del 10\%, la rentabilidad del proyecto disminuye prácticamente a cero.

El sistema ovino fue diseñado para una superficie total de pradera natural ocupada de 131 ha, según el modelo propuesto por Avendaño et al. (1978). Sin embargo, los resultados de este trabajo sugieren que el sistema mínimo rentable desde el punto de vista económico sería de 118 ha. Como el promedio de superficie de los cuatro grupos de productores analizados es menor que el área mencionada, entonces una solución viable para los productores, especialmente en los grupos de pequeños y medianos productores (cuya superficie promedio es mayor), sería a través del esquema asociativo. Considerando la dispersión en la localización de los predios (Tabla 1), la falta de capacidad de gestión individual y la alta competitividad de la industria, ambos factores detectados en el análisis FODA, se sugiere que la asociatividad adquiera la forma de sociedad anónima cerrada, tal como ocurre con otros tipos de productores en la Región del Maule. La dirección estaría a cargo de un gerente con capacidad de gestión de proyectos y nuevos negocios.

\section{Conclusiones}

En general, bajo los diferentes escenarios analizados, los resultados indican que el proyecto es rentable, considerando los principales indicadores de rentabilidad. Sin embargo, la rentabilidad del proyecto es sensible frente a una disminución en el precio de venta de los productos y a una reducción en la superficie total de pradera natural ocupada. Por lo tanto, si se asume que está dado el tamaño del sistema ovino, la variable más relevante para la proyección del negocio es el precio de los productos.

Desde un punto de vista económico, el tamaño mínimo rentable alcanzaría a una superficie de 118 ha. Sin embargo, el tamaño promedio de los predios de los cuatro grupos de productores considerados es menor que dicho tamaño mínimo rentable, lo cual sugiere que la sustentabilidad de este negocio, especialmente en los grupos de pequeños y medianos productores, estaría dada por la asociatividad, en la forma de sociedad anónima cerrada.

La viabilidad de este negocio requiere que la administración del tipo de asociatividad propuesta posea capacidad de gestión de proyectos y nuevos negocios, de manera que en el largo plazo el emprendimiento asociativo contribuya efectivamente a mejorar las condiciones de vida de los pequeños productores agropecuarios.

\section{Agradecimientos}

El autor agradece a Raúl Álvarez (Consultora PROAS), Carol Nilo (INDAP) y Jorge Zamora (Universidad de Talca), por los valiosos comentarios y sugerencias técnicas aportadas durante el desarrollo de este trabajo. Una parte de los resultados de esta investigación fue presentada como caso de estudio del proyecto Red Alfa "Micro-finance at the University" (2002-2003), co-financiado por la Comisión Europea y coordinado por la Universidad de Torino (Italia). 
AVENDAÑO, J.; FERNÁNDEZ, F.; OVALLE, C.; BLU, F. Ovinos alimentados con raciones que incluyen tagasaste (Chamaecytisus proliferus subsp. palmensis) en reemplazo de heno de alfalfa. II. Digestibilidad y consumo de nutrientes. Agricultura Técnica (Chile), v. 64, n. 3, p. 271-279, 2004.

AVENDAÑO, J.; IMBARACH, G. Efecto de la suplementación durante el periparto sobre algunos parámetros productivos y reproductivos de la oveja Suffolk-Down y su cordero en el secano interior de la provincia de Cauquenes. Agricultura Técnica (Chile), v. 62, n. 1, p. 110-120, 2002.

AVENDAÑO, J.; OVALLE, CARLOS.; ARAVENA, T. Sistema semiextensivo de producción ovina en pradera anual del secano Mediterráneo subhúmedo. Agricultura Técnica (Chile), v. 56, n. 1, p. 23-29, 1996.

AVENDAÑO, J.; OVALLE, C.; ARAVENA, T. Sistema extensivo de producción ovina en pradera anual del secano Mediterráneo subhúmedo. Agricultura Técnica (Chile), v. 54, n. 3, p. 293-301, 1994.

AVENDAÑO, J.; OVALLE, C.; FRANCO, I. Un sistema ovino propuesto para la zona centro sur del secano interior: descripción y análisis económico. Instituto de Investigaciones Agropecuarias. Estación Experimental Quilamapu. Cauquenes, Chile. Boletín Técnico, n. 13 (7 Qu), 52 p., 1978.

DÍAZ, C.; WILLIAMSON, C. Acuerdos comerciales y competitividad: evidencia del sector lácteo chileno. Revista ABANTE de Estudios en Dirección de Empresas, v. 1, n. 1, p. $58-88,1998$.

FERNÁNDEZ, F.; AVENDAÑO, J.; OVALLE, C.; FRAGA, A.; BLU, F. Ovinos alimentados con raciones que incluyen tagasaste (Chamaecytisus proliferus subsp. palmensis) en reemplazo de heno de alfalfa. I. Consumo y variaciones de peso vivo. Agricultura Técnica (Chile), v. 64 , n. 3, p. 264-270, 2004.

FIGUEROA, M. Recursos forrajeros utilizados en producción ovina. III Zona centro-sur. In: G. García (ed.) Producción Ovina. Universidad de Chile, Facultad de Ciencias Agrarias y Forestales, Santiago, Chile, 1986. p. 81-92.

FONTAINE, E. Evaluación social de proyectos. 11.ed. Santiago, Chile: Pontificia Universidad Católica de Chile, 1997. 466 p.

GALLO, C.; TADICH, E.; LANFRANCO, D.; BUNSTER, M.; BERKHOFF, F. Efectos de un programa de salud en ovinos sobre la producción cuantitativa y cualitativa de carne de cordero. Archivos de Medicina Veterinaria, v. 26, n. 21, p. 51-61, 1994.

HAYES, D.; AHN, H.; BAUMEL, P. Meat demand in South Korea: a system estimate and policy projections. Agribusiness, v. 7, n. 5, p. 433-446, 1991.

INE. 2002. Evolución, situación actual y perspectivas de la producción pecuaria nacional. 46 p. Instituto Nacional de Estadísticas. Disponible en: http://www.ine.cl/16agrope/pdf/pecuaria9702.pdf. Leído el: 2 de septiembre de 2004.

KAABIA, M.; ANGULO, A.; GIL, J. Health information and the demand for meat in Spain. European Review of Agricultural Economics, v. 28, n. 4, p. 499-517, 2001.

KOONTZ, H.; WEIHRICH, H. Administración una perspectiva global. 12.ed. México: McGraw-Hill, 2003. $804 \mathrm{p}$.

LEÓN DE CERMEÑO, J.; SCHEINER, M. Financiamiento para las micro y pequeñas empresas: algunas líneas de acción. In: LATIN AMERICA AND CARIBBEAN ECONOMIC ASSOCIATION CONGRESS, October 22-24, 1998. Proceeding. Universidad Torcuato Di Tella, Buenos Aires, Argentina. 62 p.

LOBOS, G.; SOTO, R.; ZENTENO, N.; PRIZANT, A. Análisis de eficiencia y rentabilidad económica en dos lecherías de la Región del Maule, Chile. Agricultura Técnica (Chile), v. 61, n. 3, p.3 67-378, 2001.

MONARES, A.; BUSTAMANTE, W. Situación y perspectivas de las pequeñas empresas rurales en América Latina. 2004. 48 p. Documento Preliminar - Programa de Apoyo a la Microempresa Rural de América Latina y El Caribe (PROMER).

NOVOA, R. Potencialidad y limitaciones de la agricultura del secano interior chileno. In: H. Riquelme y J. Sotomayor (eds.). SEMINARIO REALIDAD Y PERSPECTIVAS AGROPECUARIAS DEL SECANO INTERIOR. Serie Quilamapu $\mathrm{N}^{\circ} 18$. Instituto de Investigaciones Agropecuarias, Subestación Experimental Cauquenes, Cauquenes, Chile, 1989. p. 9-24.

ODEPA. Precios de productos e insumos agropecuarios. Oficina de Estudios y Políticas Agrarias. Disponible en: http://www.odepa.cl. Leído el: 15 de septiembre de 2004.

REED, A.; LEVEDAHL, W.; CLARK, S. Commercial disappearance and composite demand for food with an application to U.S. meats. Journal of Agricultural and Resource Economics, v. 28, n. 1, p. 53-70, 2003. 
ROSSON, P.; DAVIS, E.; ANGEL, A.; SEGARRA, E. Free trade impacts on US - Mexican meat trade. Agribusiness, v. 9, n. 2, p. 159-173, 1993.

SAPAG, N.; SAPAG, R. Preparación y evaluación de proyectos. 4 ed. Santiago, Chile: McGraw-Hill, 2000. $439 \mathrm{p}$.

SII. 2002. Tabla de vida útil de bienes físicos del activo inmovilizado. Servicio de Impuestos Internos. Disponible en: http://www.sii.cl/documentos/resoluciones/2002/ reso43.htm. Leído el: 5 de agosto de 2004.
VARGAS, G. Manual de criterios comunes para el control de gestión en empresas agropecuarias. 2004. 56 p. Fundación Chile y Pontificia Universidad Católica de Chile, Departamento de Economía Agraria. Santiago, Chile.

YOUNG, C. Financing the micro-scale enterprise: rural craft producers in Scotland, 1940-1914. Business History Review, v. 69, n. 3, p. 398-421, 1995.

\title{
UNDERTAKING ASSOCIATIVE SMALL HOLDING BUSINESS: ECONOMIC ANALYSIS OF THE SHEEP PRODUCTION SYSTEM, MAULE REGION, CHILE
}

\begin{abstract}
The aim of this research was to assess the economic profitability of a associative sheep husbandry business system for 20 agricultural small holdings, located in the inner unirrigated Linares area, in the Maule region, Chile. Data from the period of November 2002 until June 2003 were recollected, for characterizing the producers in four quite homogeneous sub-groups, according to the size of their herd. Profitability for a proposal system, under different price scenarios, was assessed by means of traditional indicators: Net Present Value (VAN), Internal Rate of Return (TIR) and Net Present Value Index (IVAN). The average value of the sheep herd was estimated from 0.26 to \$1.45 millions and the average value of land from 6.3 to $\$ 12.8$ millions, depending on the size of the farm (hectares). For the normal price scenario, the VAN (10.8\%) was estimated at \$ 4.12 millions, the TIR at $14.5 \%$ and the IVAN at 0.37. The main conclusion suggests that undertaking associative micro-scale enterprise can contribute to improve the life conditions of the small holding, in a better way than the average individual business.
\end{abstract}

Keywords: small holding, profitability, sheep herd, farm. 